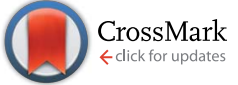

Cite this: Chem. Sci., 2017, 8, 181

\title{
Regioisomer effects of [70]fullerene mono-adduct acceptors in bulk heterojunction polymer solar cells $\dagger$
}

\author{
Tomokazu Umeyama, ${ }^{a}$ Tetsushi Miyata, ${ }^{a}$ Andreas C. Jakowetz, ${ }^{b}$ Sho Shibata, ${ }^{a}$ \\ Kei Kurotobi, ${ }^{c}$ Tomohiro Higashino, ${ }^{a}$ Tomoyuki Koganezawa, ${ }^{d}$ Masahiko Tsujimoto, ${ }^{c}$ \\ Simon Gélinas, ${ }^{\text {b }}$ Wakana Matsuda, ${ }^{a}$ Shu Seki, ${ }^{a}$ Richard H. Friend ${ }^{* b}$ \\ and Hiroshi Imahori*ac
}

\begin{abstract}
Despite numerous organic semiconductors being developed during the past decade, $\mathrm{C}_{70}$ derivatives are predominantly used as electron acceptors in efficient polymer solar cells (PSCs). However, as-prepared $\mathrm{C}_{70}$ mono-adducts intrinsically comprise regioisomers that would mask individual device performances depending on the substituent position on $C_{70}$. Herein, we separate the regioisomers of $C_{70}$ monoadducts for PSC applications for the first time. Systematic investigations of the substituent position effect using a novel symmetric $C_{70}$ mono-adduct ([70]NCMA) and a prevalent, high-performance one ([70] PCBM) reveals that we can control the structures of the blend films with conjugated polymers and thereby improve the PSC performances by regioisomer separation. Our approach demonstrates the significance of exploring the best-matching regioisomer of $\mathrm{C}_{70}$ mono-adducts with high-performance conjugated polymers, which would achieve a remarkable progress in PSC devices.
\end{abstract}

Received 5th July 2016
Accepted 6th August 2016
DOI: $10.1039 / c 6 s c 02950$ g
www.rsc.org/chemicalscience

\section{Introduction}

The demand for low-cost devices for solar energy conversion has stimulated academic and industrial researchers to develop nextgeneration photovoltaic technologies. ${ }^{1}$ Especially, polymer solar cells (PSCs) are attracting remarkable attention, because they hold promise for the realization of mechanically flexible, lightweight, large-area devices that can be fabricated by low-cost solution processes. ${ }^{2}$ The most successful PSCs to date are based on a bulk heterojunction (BHJ) structure of electron-donating low-bandgap conjugated polymers and electron-accepting fullerene derivatives. During the last decade, BHJPSCs have showed rapid progress and achieved PCEs of $6-10 \%,{ }^{3}$ mainly owing to the development of new high-performance lowbandgap polymers..$^{2,3 a, 4}$ Optimization of film structures ${ }^{3 b, c, 5}$ and developments of new buffer layer materials ${ }^{3 d, 6}$ and device structures $^{3 e, 7}$ have also contributed to the advancement.

${ }^{a}$ Department of Molecular Engineering, Graduate School of Engineering, Kyoto University, Nishikyo-ku, Kyoto, 615-8510, Japan. E-mail: imahori@scl.kyoto-u.ac.jp

${ }^{b}$ Cavendish Laboratory, University of Cambridge, JJ Thomson Avenue, Cambridge, CB3 oHE,UK.E-mail:rhf10@cam.ac.uk

'Institute for Integrated Cell-Material Sciences (WPI-iCeMS), Kyoto University, Nishikyo-ku, Kyoto 615-8510, Japan

dJapan Synchrotron Radiation Research Institute, 1-1-1, Kouto, Sayo-cho, Sayo-gun, Hyogo 679-5198, Japan

$\dagger$ Electronic supplementary information (ESI) available. CCDC 1420203-1420205. For ESI and crystallographic data in CIF or other electronic format see DOI: $10.1039 / \mathrm{c} 6 \mathrm{sc} 02950 \mathrm{~g}$
However, one lagging area is the creation of new electronaccepting materials for PSC devices. Various non-fullerene electron acceptors have been synthesized and incorporated into PSCs ${ }^{8}$ but fullerene derivatives still outperform non-fullerenes due to the small reorganization energy and thereby the superiority in the charge separation. ${ }^{9}$

In recent years, [70]fullerene derivatives are preferentially employed compared with [60]fullerene derivatives, because a higher short-circuit current density $\left(J_{\mathrm{SC}}\right)$ value can be achieved. By comparison with $\mathrm{C}_{60}$, a lower symmetry of $\mathrm{C}_{70}\left(D_{5 \mathrm{~h}} v s\right.$. $I_{\mathrm{h}}$ ) facilitates the lowest-energy transitions and a drastic increase in its absorption coefficients in the visible region is achievable. ${ }^{10}$ Extended $\pi$-conjugation of $\mathrm{C}_{70}$ could lead to a broader absorption profile as well. Currently the $\mathrm{C}_{70}$ derivative of [6,6]-phenyl- $\mathrm{C}_{61}$ butyric acid methyl ester ([60]PCBM), i.e. [70] PCBM, ${ }^{10 a}$ and its analogues are predominantly used in highperformance PSC devices in combination with low-bandgap conjugated polymers. ${ }^{3}$ However, an important aspect of $\mathrm{C}_{70}$ derivatives, which is always overlooked in PSC applications, is that as-prepared $\mathrm{C}_{70}$ mono-adducts consist of regioisomers due to the lower symmetry of rugby-ball shaped $\mathrm{C}_{70}$ over spherical $\mathrm{C}_{60}$. As contrasted with $\mathrm{C}_{60}, \mathrm{C}_{70}$ has four distinct types of $[6,6]-$ bonds ( $\alpha$-, $\beta$-, $\varepsilon^{-}$and $\kappa$-type bonds) (Fig. 1a), ${ }^{11}$ and thereby [70] fullerene derivatives including [70]PCBM have been used as a regioisomer mixture in BHJPSC devices. Considering that the miscibility with conjugated polymers and the molecular arrangement of [70]fullerene derivatives in the active layer have a large impact on charge separation and charge-transporting 
(a)

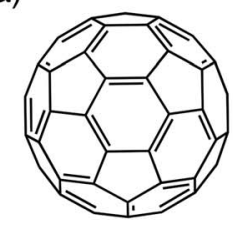

[60]fullerene

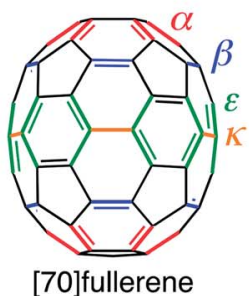

[70]fullerene (b)

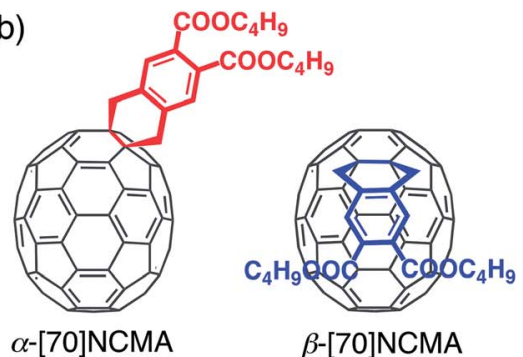

(c)

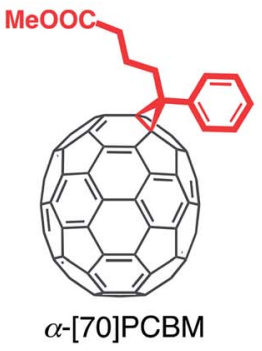

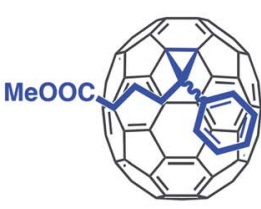

$\beta$-[70]PCBM

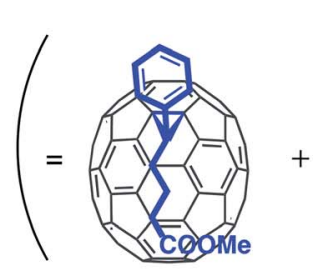

$Z-\beta-[70] P C B M$

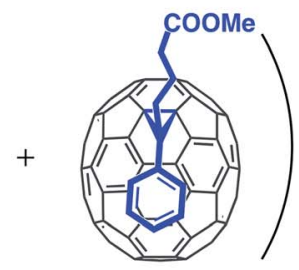

E- $\beta$-[70]PCBM

Fig. 1 (a) Structures of [60]fullerene and [70]fullerene; $\alpha^{-}, \beta-, \varepsilon^{-}$and $\kappa$-type [6,6]-bonds of [70]fullerene are represented by red, blue, green and orange lines, respectively. (b) Structures of [70]NCMA isomers. (c) Structures of [70]PCBM isomers.

properties, ${ }^{12}$ pure isomers of [70]fullerene derivatives rather than the isomer mixtures would yield a more desirable donoracceptor network toward highly efficient BHJPSCs. Indeed, the regioisomer separations of fullerene bis-adducts have been proven to exert positive effects on BHJPSCs. ${ }^{13}$

In this study, the regioisomers of [70]fullerene mono-adducts have been separated for the first time to address the pure isomer effect on the photovoltaic properties. To evaluate the substitution position effect on $\mathrm{C}_{70}$ precisely, first we designed new dihydronaphthyl-substituted [70]fullerenes with two butoxycarbonyl groups ([70]NCMA) as a simplified system. The symmetrical dihydronaphthyl group was chosen as the substituent to eliminate the plausible isomers such as enantiomers and diastereomers. ${ }^{13 d}$ Then, we have further extended this strategy to separate the regioisomers of a prevalent, high-performance [70] fullerene mono-adduct acceptor, [70]PCBM, for the first time. ${ }^{10 a}$ A systematic investigation of the substituent position effect of [70]NCMA and [70]PCBM on the film structures and photophysical and photovoltaic properties of the composite films with a widely used, high-performance conjugated polymer with an amorphous nature, poly(2,7-carbazole-alt-dithienylbenzothiadiazole) (PCDTBT), ${ }^{14}$ has been conducted.

\section{Results and discussion}

The simplified [70]fullerene mono-adduct [70]NCMA was prepared through the $[2+4]$ cycloaddition of $\mathrm{C}_{70}$ and in situ generated $o$-quinodimethane from 4,5-bis(butoxycarbonyl)benzocyclobutene. ${ }^{13 d}$ Regioisomer separation of an as-prepared [70] NCMA was performed by high-performance liquid chromatography (HPLC) equipped with a Buckyprep column specialized for the separation of fullerene regioisomers (Fig. S1a $\dagger$ ). ${ }^{13 d}$ The $\alpha$ and $\beta$-type isomers (Fig. 1b) were obtained as main products in a weight ratio of $58: 39$, and the mixture of $\alpha-[70]$ NCMA and $\beta$-[70]NCMA $(58: 39, \mathrm{w} / \mathrm{w})$ is denoted as mix-[70]NCMA. The enantiomer- and diastereomer-free structures of $\alpha$-[70]NCMA and $\beta-[70]$ NCMA allow us to investigate the regioisomer effect on photovoltaic properties precisely. Along with UV-vis absorption spectra (Fig. S2 $\dagger$ ) and other spectroscopic measurements (Experimental section in ESI $\dagger$ ), the regioisomer structures of $\alpha$-[70]NCMA and $\beta$-[70]NCMA were unambiguously confirmed by single-crystal X-ray analysis (Fig. 2a and b). The packing diagram of $\alpha-[70]$ NCMA shows an isotropically wellpacked structure (Fig. 2d-f), which is advantageous for BHJPSC device applications because it enables efficient charge transportation. On the other hand, $\beta$-[70]NCMA forms a regular porous structure along $c$-axis, whereas well-packed molecular arrays are evident along $a$ - and $b$-axes (Fig. $2 \mathrm{~g}-\mathrm{i}$ ).

A regioisomer mixture of [70]PCBM was also subjected to HPLC equipped with the Buckyprep column (Fig. S1b†). The HPLC profile shows two major fractions with a weight ratio of $12: 88$, which can be assigned to the achiral $\beta$-isomer and chiral $\alpha$-isomer of [70]PCBM, respectively (Fig. 1c). ${ }^{10 a}$ We denote here the mixture of $\beta$ - and $\alpha$-[70]PCBM $(12: 88)$ as mix-[70]PCBM. The ${ }^{1} \mathrm{H}$ NMR spectrum of $\beta$-[70]PCBM demonstrates the presence of the achiral diastereomers in which the butyric acid methyl ester group is protruding toward the equator direction (denoted as $Z-\beta-[70]$ PCBM) or the pole direction (denoted as $E-\beta$ [70]PCBM) (Fig. 1c) in the ratio of $Z$-isomer : $E$-isomer $=8: 7$ (Fig. S3 $\uparrow$ ). Furthermore, the structure of $\alpha-[70]$ PCBM was unequivocally identified by X-ray crystallography (Fig. 2c). It is evident that the $\alpha$-type $[6,6]$-bond is saturated by the addition. Although [70]PCBM is widely used in BHJPSC applications, this is the first example of an X-ray single-crystal analysis. The packing diagrams of the crystalline $\alpha$-[70]PCBM illustrate isotropically contacted fullerene cages (Fig. $2 \mathrm{j}-\mathrm{l}$ ) as is the case with $\alpha$-[70]NCMA. Single crystals of $\beta$-[70]PCBM were unable to be formed due to the presence of the diastereomers.

Electrochemical properties of the fullerene mono-adduct regioisomers were examined by cyclic voltammetry (CV) and 
(a)

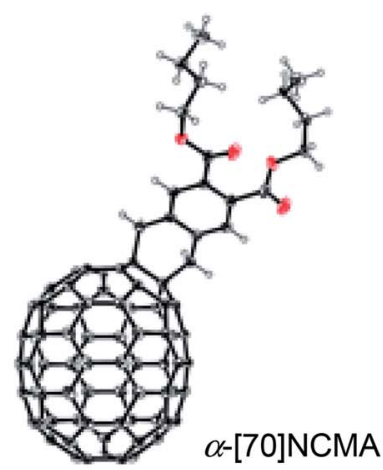

a-axis

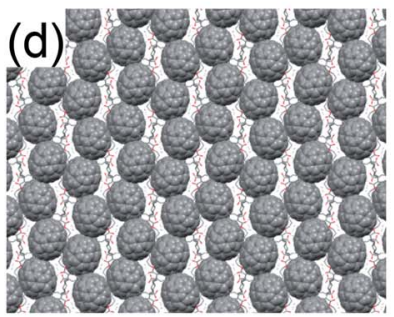

b-axis

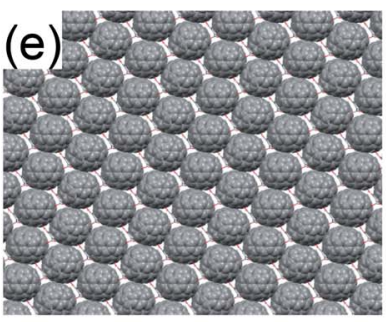

c-axis

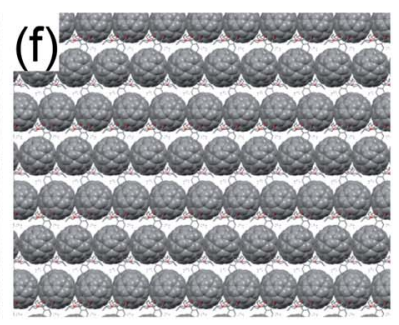

(b)

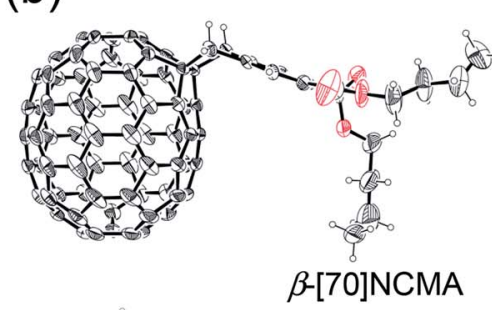

(c)

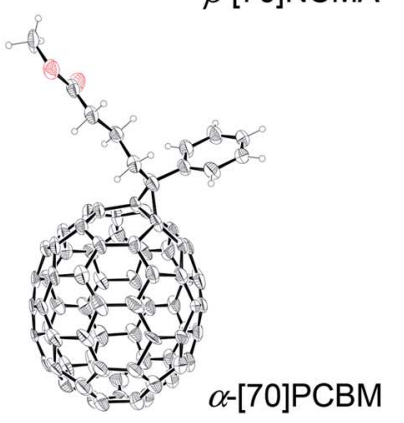

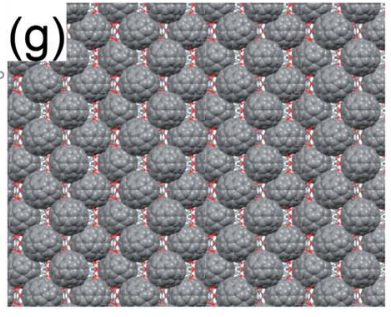
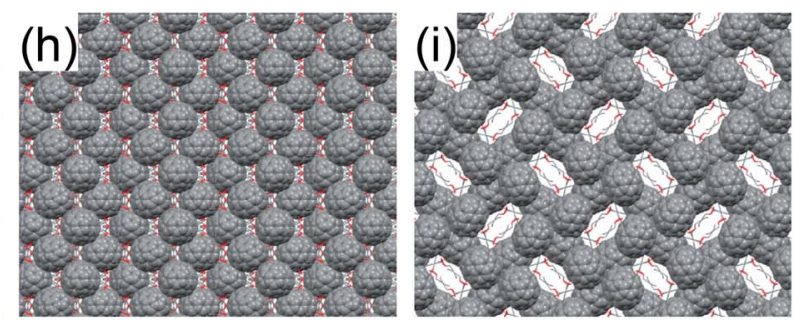
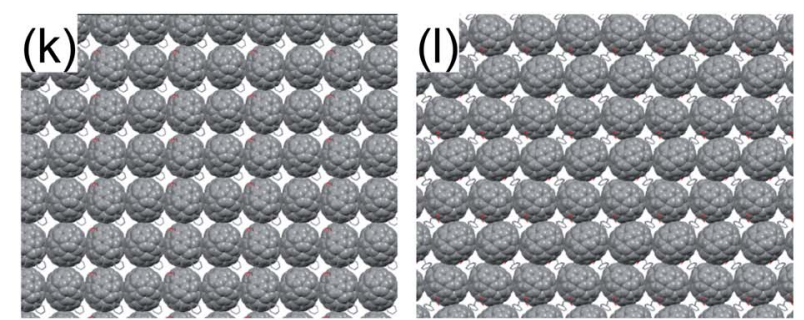

Fig. 2 X-Ray crystal structures of (a) $\alpha$-[70]NCMA, (b) $\beta$-[70]NCMA and (c) $\alpha$-[70]PCBM. Thermal ellipsoids represent 30\% probability. Minor disorder components and solvent molecules are omitted for clarity. X-Ray crystal packing structures of (d-f) $\alpha$-[70]NCMA, (g-i) $\beta$-[70]NCMA and (j-l) $\alpha$-[70]PCBM with solvent molecules along with (d, $g$ and j) $a$-axis, $(e, h$ and $k$ ) $b$-axis, and (f, i and $l) c$-axis.

differential pulse voltammetry (DPV) measurements (Fig. S4†). The first to fourth reduction potentials $\left(E_{1}-E_{4}\right)$ estimated by the peak positions in DPV are listed in Table S1. $\dagger$ The LUMO energy levels of $\alpha-[70]$ NCMA and $\alpha-[70]$ PCBM $(-3.63$ and $-3.61 \mathrm{eV})$ are slightly higher than those of the corresponding $\beta$-isomers $(-3.69$ and $-3.66 \mathrm{eV})$ (Table 1). This trend is consistent with those in the corresponding theoretical values calculated by DFT using the RB3LYP/6-31G (d) model after the geometry optimizations (Fig. S5†). These results suggest that the positions as well as the structures of addends measurably affect the electrochemical properties of [70]fullerene mono-adducts. The higher LUMO levels of the $\alpha$-isomers would be favorable for the application as electron acceptors in BHJPSCs to improve the $V_{\mathrm{OC}}$ value because $V_{\mathrm{OC}}$ of a BHJPSC device is dependent on the energy difference between HOMO of the donor and LUMO of the acceptor. ${ }^{15}$ Thermal gravimetric analysis (TGA, Fig. S6 $\dagger$ ) and differential scanning calorimetry (DSC, Fig. S7 $\dagger$ ) measurements revealed sufficient thermal stabilities of the regioisomerically pure fullerene mono-adducts for PSC device applications.

BHJPSC devices with a configuration of ITO/PEDOT:PSS/ PCDTBT : fullerene/ $\mathrm{TiO}_{x} / \mathrm{Al}$ were fabricated to evaluate the effect of the fullerene mono-adduct regioisomers on their photovoltaic performances. The weight ratios of [PCDTBT] : [fullerene] were optimized to achieve the highest PCE values and the optimized ratios are listed in Table 1. Current density-voltage curves averaged from ten independent PSC devices are shown in Fig. 3. The averaged photovoltaic parameters as well as those of the best-performing devices are listed in Table 1. In addition, the averaged parameters with variation values are also summarized in Table S2. $\dagger$ The variations in all photovoltaic parameters are substantially small and thereby corroborate the statistical significance of the averaged values. As shown in Fig. 3a and Table 1, the regioisomer pure $\beta$-[70]NCMA-based device exhibits slightly inferior PSC device performance (PCE $=2.44 \%$ ) to the mix-[70]NCMA-based one $(\mathrm{PCE}=2.71 \%)$, whereas the PCE $(4.04 \%)$ of the $\alpha$-[70]NCMAbased one is significantly higher than the others. All photovoltaic parameters, i.e. short-circuit current density $\left(J_{\mathrm{sC}}\right)$, opencircuit voltage $\left(V_{\mathrm{OC}}\right)$ and fill factor $(\mathrm{FF})$ of $\alpha$-[70]NCMA are higher than those of $\beta-[70]$ NCMA and mix-[70]NCMA. To the best of our knowledge, this is the first example of the performance improvement of the BHJPSC devices by regioisomer separation 
Table 1 LUMO energy levels and microwave conductivities of fullerenes and device parameters, ${ }^{a}$ correlation lengths, and $\chi$ values of PCDTBT : fullerene blend films

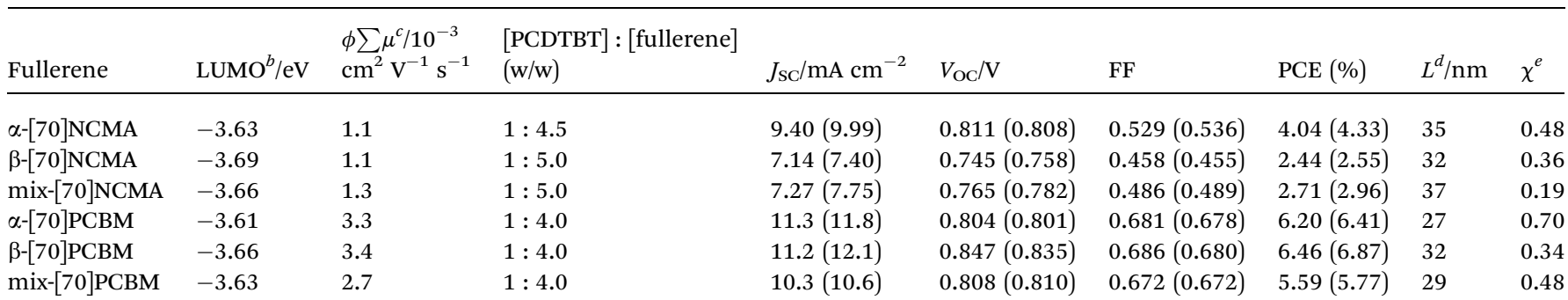

${ }^{a}$ The photovoltaic parameters were averaged from ten independent solar cells. The parameters of the best-performing devices are also shown in parentheses. ${ }^{b} \mathrm{LUMO} / \mathrm{eV}=-\left(4.8-E_{1}\right)$, where $E_{1}$ values are the first reduction potentials determined by DPV measurements. ${ }^{c}$ Maximum values of transient conductivities determined by flash-photolysis TRMC method upon photoirradiation at $355 \mathrm{~nm}$ with a photon density of $4.6 \times 10^{15}$ photons $\mathrm{cm}^{-2}$. ${ }^{d}$ Correlation lengths based on the Debye-Anderson-Brumberger model from one-dimensional GISAXS profiles. ${ }^{e}$ Exponent of the power-law fits in TA.

from the isomer mixture of [70]fullerene mono-adducts. Reflecting the higher $J_{\mathrm{SC}}$ in the device with $\alpha$-[70]NCMA $(9.40$ $\left.\mathrm{mA} \mathrm{cm}{ }^{-2}\right)$ than those of $\beta$-[70]NCMA $\left(7.14 \mathrm{~mA} \mathrm{~cm}^{-2}\right)$ and mix[70]NCMA $\left(7.27 \mathrm{~mA} \mathrm{~cm}^{-2}\right)$, the $\alpha-[70]$ NCMA-based device exhibits higher incident photon-to-current efficiency (IPCE) in the whole visible region (Fig. S8a†).

The BHJPSCs based on [70]PCBM isomers reveal averaged PCE values of $5.59-6.46 \%$ (Fig. $3 \mathrm{~b}$ and Table 1), which are comparable with previously reported ones of PCDTBT:[70] PCBM-based devices $(5-7 \%)^{14,16}$ and significantly higher than those of [70]NCMA isomers (2.44-4.04\%). More importantly, the regioisomer-pure $\alpha$-[70]PCBM- and $\beta$-[70]PCBM-based devices ( $\mathrm{PCE}=6.20$ and $6.46 \%$, respectively) considerably outperform the mix-[70]PCBM-based one ( $\mathrm{PCE}=5.59 \%)$. The highest PCE is attained by the $\beta$-isomer for [70]PCBM, whereas the $\alpha$-isomer shows the highest PCE for [70]NCMA. The best-matching substituent position compatible with a conjugated polymer strongly depends on the adduct structure on $\mathrm{C}_{70}$. The $J_{\mathrm{SC}}$ of the devices with $\alpha$-[70]PCBM $\left(U_{\mathrm{SC}}=11.3 \mathrm{~mA} \mathrm{~cm}^{-2}\right)$ and $\beta$-[70]PCBM $\left(J_{\mathrm{SC}}=11.2 \mathrm{~mA} \mathrm{~cm}^{-2}\right)$ are higher than that of mix-[70]PCBM $\left(J_{\text {sC }}=10.3 \mathrm{~mA} \mathrm{~cm}^{-2}\right.$ ) (Table 1 and Fig. S8b $\dagger$ ). It is noteworthy that the regioisomer effect on PCE is more significant in [70]
NCMA isomers than [70]PCBM ones. The enantiomer- and diastereomer-free structure of [70]NCMA may reinforce the influence of the regioisomer effect on photovoltaic properties.

Comparing the photovoltaic parameters listed in Table 1, the $J_{\mathrm{SC}}$ are the most pronounced factor for leading to a difference in the PCE. In PSC devices, the conversion of light-energy to electricity involves the following fundamental steps: (1) lightharvesting, (2) exciton collection at the polymer-fullerene interface, (3) exciton dissociation, and (4) charge collection. The $J_{\mathrm{SC}}$ of a solar cell depends on the efficiencies corresponding to these four steps. With regard to step 1, light-harvesting efficiencies of the [70]NCMA and [70]PCBM isomers (Fig. S2 $\dagger$ ) and their composite films with PCDTBT (Fig. S9†) are comparable, indicating that this step does not make a significant difference in the $J_{\mathrm{sc}}$. Steady-state photoluminescence measurements for the polymer quenching suggest the exciton collection efficiencies (step 2) in the polymer domains are almost unity in all devices (Fig. S10 $\dagger$ ). Due to the amorphous nature of PCDTBT as well as the high composition ratio of the fullerenes ([PCDTBT] : [fullerene] $=1: 4-5$, Table 1 ), essentially all the polymer in the BHJ films is well mixed with at least a certain fraction of the fullerene on a scale of the exciton diffusion (a)

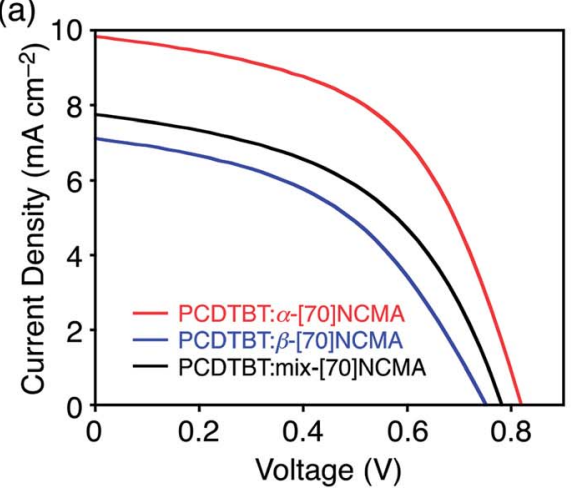

(b)

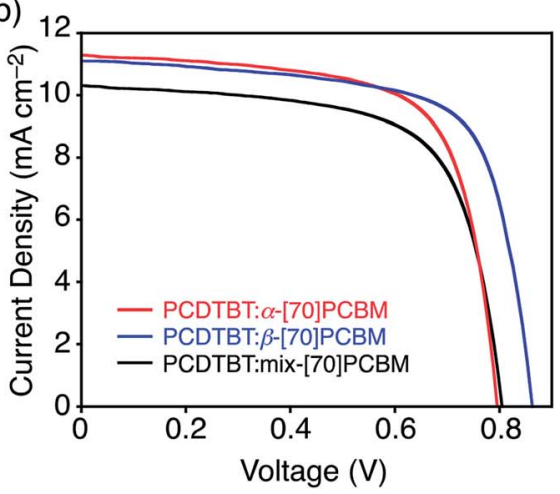

Fig. 3 Current density-voltage curves averaged from ten independent PSC devices based on (a) PCDTBT: $\alpha$-[70]NCMA (red), PCDTBT : $\beta$-[70] NCMA (blue) and PCDTBT : mix-[70]NCMA (black), and (b) PCDTBT : $\alpha$-[70]PCBM (red), PCDTBT : $\beta$-[70]PCBM (blue) and PCDTBT : mix-[70] PCBM (black) films. 

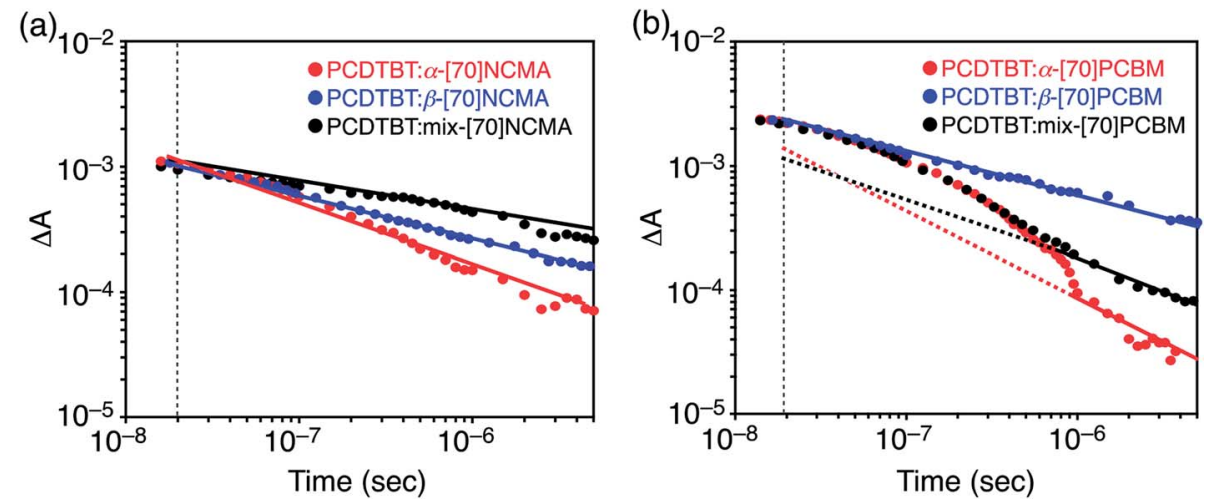

Fig. 4 Transient absorption decay kinetics of (a) PCDTBT : $\alpha$-[70]NCMA (red), PCDTBT: $\beta$-[70]NCMA (blue) and PCDTBT : mix-[70]NCMA (black), and (b) PCDTBT : $\alpha$-[70]PCBM (red), PCDTBT : $\beta$-[70]PCBM (blue) and PCDTBT : mix-[70]PCBM (black) films. Excitation and probe wavelengths are 532 and $970-1000 \mathrm{~nm}$, respectively. The $\Delta A$ signal intensities are normalized at 20 ns. Fitting lines with power-law decays (straight lines on the log-log plot) are also shown. Dotted lines for PCDTBT : $\alpha$-[70]PCBM and PCDTBT : mix-[70]PCBM in (b) are extrapolated from the power-law decays. The vertical gray dotted lines are located at $20 \mathrm{~ns}$.

length $(<10 \mathrm{~nm}) \cdot{ }^{17}$ Furthermore, grazing-incidence small-angle X-ray scattering (GISAXS) measurements were conducted to examine the statistically averaged fullerene domain sizes (Fig. S11†), ${ }^{18}$ which affect the collection efficiency of the excitons formed in the fullerene domains. The correlation lengths $(L)$ based on the Debye-Anderson-Brumberger (DAB) model ${ }^{18}$ reflect the average fullerene domain size (Fig. S12 $\dagger$ ), which are listed in Table 1. Whereas the weight ratios of [PCDTBT] : [fullerene] were respectively optimized to achieve the highest PCE values, the domain sizes of the fullerene mono-adducts are large $(\geq 27 \mathrm{~nm}$ ) compared to the exciton diffusion lengths of fullerene derivatives, which are limited to $c a .5 \mathrm{~nm} .{ }^{19}$ Such large fullerene domains may decrease the collection efficiencies of excitons generated in the fullerene domains. Although the fullerene domain sizes show no clear relationship to the $J_{\mathrm{SC}}$ of the PSC devices (Table 1), the larger fullerene domain sizes in PCDTBT : [70]NCMA isomers (32-37 nm) than PCDTBT : [70] PCBM isomers (27-32 nm) may be one of the factors that the $J_{\mathrm{SC}}$ of the [70]NCMA isomer-based devices are lower than those of the [70]PCBM isomer-based ones. ${ }^{20}$ In contrast, atomic force microscopy (AFM, Fig. S13 $\dagger$ ) and two-dimensional grazingincidence wide-angle X-ray scattering (GIWAXS, Fig. S14 $\dagger$ ) measurements displayed no significant differences in the PCDTBT : fullerene composite films, reflecting the amorphous nature of PCDTBT.

Electron and hole mobilities of fullerene and polymer domains in blend films exert an influence on the charge collection efficiency (step 4). To estimate the intrinsic electron transporting abilities of the fullerene materials, we measured the flash-photolysis time-resolved microwave conductivity (TRMC) of the fullerene neat films. ${ }^{21,22}$ Upon exposure to a laser pulse with an excitation wavelength of $355 \mathrm{~nm}$, all samples reveal a rise of the transient conductivity $\left(\phi \sum \mu\right)$, in which $\phi$ is the quantum efficiency of charge separation (CS) and $\sum \mu$ is the sum of the mobilities of all the transient charge carriers (Table 1 and Fig. S15 $\dagger$ ). The major charge carriers stem from electrons in all the fullerene films. Apparently, the differences in the regioisomers lead to insignificant effects on the $\phi \sum \mu$ values $\left((1.1-1.3) \times 10^{-9} \mathrm{~cm}^{2} \mathrm{~V}^{-1} \mathrm{~s}^{-1}\right.$ for the [70]NCMA isomers and $(2.7-3.4) \times 10^{-9} \mathrm{~cm}^{2} \mathrm{~V}^{-1} \mathrm{~s}^{-1}$ for the [70]PCBM isomers) and therefore the electron mobilities, supposing that the $\phi$ values are comparable in the fullerene neat films. Nevertheless, relatively large $\phi \sum \mu$ values of the [70]PCBM isomers compared to the [70]NCMA ones may also be another reason for the fact that the [70]PCBM isomer-based devices outperform the [70]NCMA isomer-based ones.

To shed more light onto charge collection efficiencies (step 4), we conducted transient absorption (TA) measurements of the PCDTBT : fullerene composite films. The TA decay kinetics obtained by the excitation at $532 \mathrm{~nm}$ for the PCDTBT : fullerene composite films are shown in Fig. 4 . The presented data (>10 ns) shown between 970 and $1000 \mathrm{~nm}$ represents the hole polaron population, which decays by a non-geminate recombination process. ${ }^{23}$ PCDTBT : [70]NCMA isomer films reveal power-law decay kinetics $\left(\Delta A \propto t^{-\chi}\right)$, i.e. straight lines on the log-log plot, over the whole time range (Fig. 4a). The power-law like decays are characteristic of non-geminate recombination of dissociated free charge carriers. ${ }^{\mathbf{1 2 , 2 4}}$ In addition, $\chi$ is known to correlate with polaron trapping in a distribution of energetic traps. ${ }^{23 a, 25}$ The highest $J_{\mathrm{SC}}$ of the PCDTBT : $\alpha$-[70]NCMA film $(9.40 \mathrm{~mA}$ $\mathrm{cm}^{-2}$ ) among the PCDTBT : [70]NCMA isomer films is consistent with the highest $\chi(0.48)$. This result arises from the relatively isotropic packing structure of fullerene molecules in PCDTBT : $\alpha$-[70]NCMA (Fig. 2d-f) which is favorable for efficient charge transportation and thereby increases the charge collection efficiency (step 4). The holey packing structure of $\beta$-[70] NCMA observed in the single-crystal structure (Fig. 2i) may also be formed in the PCDTBT : $\beta$-[70]NCMA blend film, which may act as a trapping site of the fullerene polarons. On the other hand, the emergence of the monoexponential-like decay within the first $1 \mu$ s only in PCDTBT : $\alpha$-[70]PCBM and PCDTBT : mix[70]PCBM suggests the lower cohesive nature of $\alpha$-[70]PCBM than $\beta$-[70]PCBM and [70]NCMA isomers. This result is consistent with the fact that the sizes of fullerene-rich domains in PCDTBT $: \alpha-[70]$ PCBM and PCDTBT : mix-[70]PCBM (27 and $29 \mathrm{~nm}$, respectively) estimated by the GISAXS measurements are 
smaller than those in PCDTBT : $\beta$-[70]PCBM and PCDTBT : [70] NCMA isomers $(>32 \mathrm{~nm})$. In contrast to $\beta$-[70]PCBM, the diastereomer-free structure of $\alpha$-[70]PCBM may enable the isotropically well-packed fullerene alignments as observed in the single crystal structure (Fig. 2j-l) even in the composite film with PCDTBT, and lead to stronger delocalization on the fullerenes. This is reflected in the highest $\chi(0.70)$ of the PCDTBT : $\alpha-[70]$ PCBM films in the time region of $>1 \mu \mathrm{s}$.

Irrespective of the lower $\chi, J_{\mathrm{SC}}$ of the device with PCDTBT : $\beta-[70]$ PCBM $\left(11.3 \mathrm{~mA} \mathrm{~cm}{ }^{-2}\right)$ is comparable to that with PCDTBT : $\alpha-[70]$ PCBM $\left(11.2 \mathrm{~mA} \mathrm{~cm}^{-2}\right)$. This indicates the higher charge dissociation efficiency (step 3) of PCDTBT : $\beta$-[70]PCBM, that is, the geminate recombination from the tightly bound charge-transfer (CT) state occurs less frequently in PCDTBT : $\beta-[70]$ PCBM than in PCDTBT $: \alpha-[70]$ PCBM due to the higher cohesive nature of $\beta$-[70]PCBM. ${ }^{26}$ This suppressed geminate recombination in PCDTBT: $\beta-[70]$ PCBM may also explain the higher $V_{\mathrm{OC}}$ of the PCDTBT: $\beta$-[70]PCBM-based device (Table 1), whereas the LUMO level of $\alpha$-[70]PCBM is most elevated (Table 1). ${ }^{15}$ Additionally, overall higher $J_{\mathrm{SC}}$ in PCDTBT : [70]PCBM blend films than in PCDTBT : [70]NCMA films may result from the higher exciton dissociation efficiency (step 3), as well as the higher exciton collection efficiency in the fullerene domains (step 2, Fig. S12†).

\section{Conclusion}

In summary, we have successfully established the close relationship between the film structures, photophysics, and photovoltaic properties of the isomer-pure [70]fullerene monoadducts for the first time. These results demonstrate that the isolation of the pure regioisomers of [70]fullerene monoadducts by using HPLC specialized for fullerene derivatives, is of extreme value to control the structures of the blend films with conjugated polymers, thereby improving the BHJPSC performances. Unfortunately, the enantiomers of $\alpha$-[70]PCBM and diastereomers of $\beta$-[70]PCBM were unable to be separated even by the special HPLC technique. Nevertheless, considering that the enantiomer- and diastereomer-free [70]NCMA showed more pronounced regioisomer effects on photovoltaic properties than [70]PCBM, the enantiomer separation of $\alpha-[70]$ PCBM and the diastereomer separation of $\beta$-[70]PCBM could further improve the device performances. Our approach will drive material researchers to explore the best-matching pure regioisomer of well-tailored [70]fullerene mono-adducts with high-performance conjugated polymers, which can bring about a remarkable progress in PSC devices.

\section{Experimental section}

\section{Instruments}

Single-crystal X-ray diffraction analysis of $\alpha-[70]$ NCMA was conducted at $-150{ }^{\circ} \mathrm{C}$ with a Rigaku Saturn 70 by using graphitemonochromated Mo-K $\alpha$ radiation $(\lambda=0.71069 \AA)$. The analyses of $\beta$-[70]NCMA and $\alpha$-[70]PCBM were performed at $-180^{\circ} \mathrm{C}$ with a Rigaku XtaLAB P200 apparatus using the two-dimensional detector PILATUS $100 \mathrm{~K} / \mathrm{R}$ with $\mathrm{Cu}-\mathrm{K} \alpha$ radiation $(\lambda=1.54187 \AA)$.
The structures were solved by direct method (SHELXS-2013). CCDC $1420205(\alpha-[70]$ NCMA), $1420204(\beta-[70]$ NCMA) and $1420203(\alpha-[70]$ PCBM $)$ contain the supplementary crystallographic data for this paper.

Photocurrent-voltage characteristics were measured by a Keithley 2400 SourceMeter under an argon atmosphere and simulated solar light (100 $\mathrm{mW} \mathrm{cm}^{-2}$, AM1.5) with an OTENTOSUN III solar simulator (Bunkoukeiki). Photocurrent action spectra were recorded with a CEP-2000RR (Bunkoukeiki). Current-voltage characteristics of the electron- and hole-only devices for space-charge-limited current (SCLC) measurements were conducted using a Keithley 2400 SourceMeter under an argon atmosphere.

\section{Materials}

$\mathrm{C}_{70}(98.0 \%)$ was obtained from MTR Ltd. and used as-received. 4,5-Bis(butoxycarbonyl)benzocyclobutene was prepared according to the reported procedure. ${ }^{13 d}$ Phenyl $\mathrm{C}_{71}$-butyric acid methyl ester ([70]PCBM, >99.0\%) was purchased from American Dye Source, Inc. All other solvents and chemicals were of reagentgrade quality, purchased commercially, and used without further purification unless otherwise noted. Thin layer chromatography (TLC) and column chromatography were performed with Silica gel $60 \mathrm{~F}_{254}$ (Merck) and SiliaFlash F60 (230-400 mesh; SiliCycle Inc.), respectively. Details of synthesis of [70]NCMA and isomer separations of [70]NCMA and [70]PCBM are described in ESI. $\dagger$

\section{Device fabrication}

Indium tin oxide (ITO) on a glass substrate with a sheet resistance of $5 \Omega$ sq. $^{-1}$ (Geomatec) was used. The substrates were sonicated consecutively with water, chloroform, acetone, semico clean (Furuuchi Chemical), 2-propanol and ethanol. After blow-drying and UV-ozone treatment, the substrates were spincoated at $1000 \mathrm{rpm}$ and then $4000 \mathrm{rpm}$ with poly(ethylene dioxythiophene) doped with polystyrene sulfonic acid (PEDOT:PSS, Clevios P) and dried at $200^{\circ} \mathrm{C}$. For the fabrication of the active layer with bulk heterojunction structure, a mixed solution of PCDTBT $\left(7 \mathrm{mg} \mathrm{mL}^{-1}\right) /$ fullerene $(\alpha-[70]$ NCMA: 31.5 $\mathrm{mg} \mathrm{mL}{ }^{-1}, \beta-[70]$ NCMA: $35 \mathrm{mg} \mathrm{mL}^{-1}$, mix-[70]NCMA: $35 \mathrm{mg}$ $\mathrm{mL}^{-1}$, [70]PCBM isomers: $28 \mathrm{mg} \mathrm{mL}{ }^{-1}$ ) in $o$-dichlorobenzene were spin-coated at $1000 \mathrm{rpm}$ onto the ITO/PEDOT:PSS. The film thicknesses of the active layers are $c a .110 \mathrm{~nm}$. After spin coating, thermal annealing of the active layer was conducted at $70{ }^{\circ} \mathrm{C}$ for $60 \mathrm{~min}$. For the fabrication of the buffer layer, a solution of titanium isopropoxide in ethanol was spin-coated at $4000 \mathrm{rpm}$ onto the ITO/PEDOT:PSS/PCDTBT : fullerene. Then, Al was deposited to obtain the PSC device denoted as ITO/ PEDOT:PSS/PCDTBT : fullerene/TiO $/$ /Al.

The electron-only devices for the SCLC measurements were fabricated as follows. A $50 \mathrm{~nm}$ Al film was first thermally deposited onto the glass substrate. The PCDTBT : fullerene blend film with the same ratio as in the PSC device was spin-coated at $800 \mathrm{rpm}$. Then, the active layer was capped by a $100 \mathrm{~nm} \mathrm{Al}$ electrode. Meanwhile, the SCLC measurements for hole mobilities were conducted using the device with the configuration of ITO/PEDOT:PSS/PCDTBT : fullerene/Au $(100 \mathrm{~nm})$. 


\section{Transient absorption spectroscopy}

The pump pulses (at $532 \mathrm{~nm}$ ) were generated using the second harmonic output of a Q-switched $\mathrm{Nd}: \mathrm{YVO}_{4}$ laser (AOT-YVO25QSPX, Advanced Optical Technologies Ltd.). The broadband probe pulses were generated using a home-built non-collinear optical parametric amplifier (NOPA) seeded with a portion of the output of a Ti:sapphire amplifier system (Solstice, SpectraPhysics) operating at $1 \mathrm{kHz}$. The resulting NOPA output was split into a probe and a reference beam which allows accounting for shot-to-shot laser fluctuations. The pump and probe beams were focused and overlapped on the film and their time delay was electronically set using a time delay generator (DG535, Stanford Research Systems) while the reference beam was passed through the sample at a spot unaffected by the pump. The transmitted probe and reference beams were dispersed in a spectrometer (Shamrock SR-303i, Andor Technology) and detected using a pair of 16 bit, 512-pixel linear InGaAs image sensors (Hamamatsu) connected to a custom-built board enabling data acquisition at $1 \mathrm{kHz}$ (Entwicklungsbüro Stresing).

\section{Acknowledgements}

This work is supported by New Energy and Industrial Technology Development Organization (NEDO), Grand-in-Aid (No. 25220801 to H. I.), the Engineering and Physical Sciences Research Council (EPSRC), and the Winton Programme (Cambridge) for the Physics of Sustainability. The synchrotron radiation experiments for GISAXS and GIWAXS measurements were performed at the BU46XU of SPring-8 with the approval of the Japan Synchrotron Radiation Research Institute (JASRI) (Proposal No. 2014B1596). The authors thank Prof. Atsuhiro Osuka and Prof. Takayuki Tanaka (Kyoto University) for singlecrystal X-ray diffraction analyses. A.C.J. thanks the University of Cambridge for funding (CHESS).

\section{Notes and references}

1 P. K. Nayak and D. Cahen, Adv. Mater., 2014, 26, 1622.

2 (a) L. Dou, J. You, Z. Hong, Z. Xu, G. Li, R. A. Street and Y. Yang, Adv. Mater., 2013, 25, 6642; (b) T. Umeyama and H. Imahori, J. Mater. Chem. A, 2014, 2, 11545; (c) K. A. Mazzio and C. K. Luscombe, Chem. Soc. Rev., 2015, 44, 78.

3 (a) Z. He, B. Xiao, F. Liu, H. Wu, Y. Yang, S. Xiao, C. Wang, T. P. Russell and Y. Cao, Nat. Photonics, 2015, 9, 174; (b) Y. Liu, J. Zhao, Z. Li, C. Mu, W. Ma, H. Hu, K. Jiang, H. Lin, H. Ade and H. Yan, Nat. Commun., 2015, 5, 5293; (c) V. Vohra, K. Kawashima, T. Kakara, T. Koganezawa, I. Osaka, K. Takimiya and H. Murata, Nat. Photonics, 2015, 9, 403; (d) S.-H. Liao, H.-J. Jhuo, P.-N. Yeh, Y.-S. Cheng, Y.-L. Li, Y.-H. Lee, S. Sharma and S.-A. Chen, Sci. Rep., 2014, 4, 6813; (e) J. Zhang, Y. Zhang, J. Fang, K. Lu, Z. Wang, W. Ma and Z. Wei, J. Am. Chem. Soc., 2015, 137, 8176.

4 H. Zhou, L. Yang and W. You, Macromolecules, 2012, 45, 607.
5 M. T. Dang, L. Hirsch, G. Wantz and J. D. Wuest, Chem. Rev., 2013, 113, 3734.

6 Z. A. Page, Y. Liu, V. V. Duzhko, T. P. Russell and T. Emrick, Science, 2014, 346, 441.

7 (a) L. Lu, M. A. Kelly, W. You and L. Yu, Nat. Photonics, 2015, 9, 491; (b) I. Litzov and C. J. Brabec, Materials, 2013, 6, 5796. 8 (a) C. L. Chochos, N. Tagmatarchis and V. G. Gregoriou, RSC Adv., 2013, 3, 7160; (b) Y. Zhong, M. T. Trinh, R. Chen, G. E. Purdum, P. P. Khlyabich, M. Sezen, S. Oh, H. Zhu, B. Fowler, B. Zhang, W. Wang, C.-Y. Nam, M. Y. Sfeir, C. T. Black, M. L. Steigerwald, Y.-L. Loo, F. Ng, X.-Y. Zhu and C. Nuckolls, Nat. Commun., 2015, 6, 8242; (c) Y.-J. Hwang, B. A. E. Courtright, A. S. Ferreira, S. H. Tolbert and S. A. Jenekhe, Adv. Mater., 2015, 27, 4578.

9 (a) H. Imahori, Bull. Chem. Soc. Jpn., 2007, 80, 621; (b) B. M. Savoie, A. Rao, A. A. Bakulin, S. Geélinas, B. Movaghar, R. H. Friend, T. J. Marks and M. A. Ratner, J. Am. Chem. Soc., 2014, 136, 2876.

10 (a) M. M. Wienk, J. M. Kroon, W. J. H. Verhees, J. Knol, J. C. Hummelen, P. A. van Hal and R. A. J. Janssen, Angew. Chem., Int. Ed., 2003, 42, 3371; (b) X. Guo, C. H. Cui, M. J. Zhang, L. J. Huo, Y. Huang, J. H. Hou and Y. Li, Energy Environ. Sci., 2012, 5, 7943.

11 (a) A. Hermann, F. Diederich, C. Thilgen, H.-U. ter Meer and W. H. Meuller, Helv. Chim. Acta, 1994, 77, 1689; (b) M. R. Cerón, M. Izquierdo, A. Aghabali, J. A. Valdez, K. B. Ghiassi, M. M. Olmstead, A. L. Balch, F. Wudl and L. Echegoyen, J. Am. Chem. Soc., 2015, 137, 7502.

12 (a) E. T. Hoke, K. Vandewal, J. A. Bartelt, W. R. Mateker, J. D. Douglas, R. Noriega, K. R. Graham, J. M. J. Fréchet, A. Salleo and M. D. McGehee, Adv. Energy Mater., 2013, 3, 220; (b) S. Shoaee, S. Subramaniyan, H. Xin, C. Keiderling, P. S. Tuladhar, F. Jamieson, S. A. Jenekhe and J. R. Durrant, Adv. Funct. Mater., 2013, 23, 3286.

13 (a) S. Kitaura, K. Kurotobi, M. Sato, Y. Takano, T. Umeyama and H. Imahori, Chem. Commun., 2012, 48, 8550; (b) D. S. Sabirov, J. Phys. Chem. C, 2013, 117, 9148; (c) X. Meng, G. Zhao, Q. Xu, Z. a. Tan, Z. Zhang, L. Jiang, C. Shu, C. Wang and Y. Li, Adv. Funct. Mater., 2014, 24, 158; (d) R. Tao, T. Umeyama, K. Kurotobi and H. Imahori, ACS Appl. Mater. Interfaces, 2014, 6, 17313.

14 S. Beaupré and M. Leclerc, J. Mater. Chem. A, 2013, 1, 11097. 15 B. Qi and J. Wang, J. Mater. Chem., 2012, 22, 24315.

16 T. Umeyama, Y. Watanabe, E. Douvogianni and H. Imahori, J. Phys. Chem. C, 2013, 117, 21148.

17 O. V. Mikhnenko, H. Azimi, M. Scharber, M. Morana, P. W. M. Blomad and M. A. Loi, Energy Environ. Sci., 2012, 5, 6960.

18 (a) H.-C. Liao, C.-S. Tsao, T.-H. Lin, C.-M. Chuang, C.-Y. Chen, U.-S. Jeng, C.-H. Su, Y.-F. Chen and W.-F. Su, J. Am. Chem. Soc., 2011, 133, 13064; (b) Y.-C. Huang, C.-S. Tsao, C.-M. Chuang, C.-H. Lee, F.-H. Hsu, H.-C. Cha, C.-Y. Chen, T.-H. Lin, C.-J. Su, U.-S. Jeng and W.-F. Su, J. Phys. Chem. C, 2012, 116, 10238; (c) M.-S. Su, C.-Y. Kuo, M.-C. Yuan, U.-S. Jeng, C.-J. Su and K.-H. Wei, Adv. Mater., 2011, 23, 3315; (d) P. Debye, R. Anderson and H. Brumberger, J. Appl. Phys., 1957, 28, 679. 
19 (a) G. F. Burkhard, E. T. Hoke, S. R. Scully and M. D. McGehee, Nano Lett., 2009, 9, 4037; (b) S. Cook, A. Furube, R. Katoh and L. Han, Chem. Phys. Lett., 2009, 478, 33.

20 We utilized an additive, 1,8-diiodooctane (DIO), for the fabrication of the device based on PCDTBT : $\alpha-[70]$ NCMA with an attempt to further optimize the film structures and thereby improve the device performances. However, the averaged PCE values of more than ten independent devices were $3.78 \%$ ( 1 vol\% DIO) and $3.82 \%$ (3 vol\% DIO), which are slightly lower than that of the device without DIO (4.04\%).

21 T. Umeyama, N. Tezuka, S. Seki, Y. Matano, M. Nishi, K. Hirao, H. Lehtivuori, N. V. Tkachenko, H. Lemmetyinen, Y. Nakao, S. Sakaki and H. Imahori, Adv. Mater., 2010, 22, 1767.

22 We also attempted to evaluate the electron- and holemobilities ( $\mu_{\mathrm{e}}$ and $\mu_{\mathrm{h}}$, respectively) of the PCDTBT : fullerene blend films by space charge limited current (SCLC) measurements. However, despite the careful optimization of the PCDTBT : fullerene ratio in the PSC devices, the mobility balances, i.e. $\mu_{\mathrm{e}} / \mu_{\mathrm{h}}$, were very poor due to the much lower $\mu_{\mathrm{h}}$ values $\left(\mu_{\mathrm{e}}=(0.1-3) \times 10^{-3} \mathrm{~cm}^{2} \mathrm{~V}^{-1} \mathrm{~s}^{-1}\right.$ and $\mu_{\mathrm{h}}<10^{-6} \mathrm{~cm}^{2}$ $\left.\mathrm{V}^{-1} \mathrm{~s}^{-1}\right)$. This result conflicts with the well-known fact that the balanced charge mobilities make a positive effect on the device performances. Achievements and accurate measurements of SCLC require various conditions such as an ohmic contact between the electrode and the semiconductor, lower number of film free charge carriers than the injected carriers, and the formation of charge transport pathways throughout the film thickness. It may be difficult to fulfill all the required conditions to measure
SCLC precisely in the PCDTBT : fullerene blend films, resulting in mobility values with low reliability. Especially, the construction of the hole transport pathway is prohibitively difficult due to the low content of PCDTBT (Table 1). As references for the effect of mobility balances on PSCs, see; (a) H. Ito, T. Iwata, S. Watanabe and S. Kuroda, Appl. Phys. Express, 2013, 6, 051601; (b) T. Mikie, A. Saeki, H. Masuda, N. Ikuma, K. Kokubo and S. Seki, J. Mater. Chem. A, 2015, 3, 1152; (c) J. D. Kotlarski and P. W. M. Blom, Appl. Phys. Lett., 2012, 100, 013306.

23 (a) F. Etzold, I. A. Howard, R. Mauer, M. Meister, T.-D. Kim, K.-S. Lee, N. S. Baek and F. Laquai, J. Am. Chem. Soc., 2011, 133, 9469; (b) N. Banerji, S. Cowan, M. Leclerc, E. Vauthey and A. J. Heeger, J. Am. Chem. Soc., 2010, 132, 17459.

24 (a) H. Ohkita, S. Cook, Y. Astuti, W. Duffy, S. Tierney, W. Zhang, M. Heeney, I. McCulloch, J. Nelson, D. D. C. Bradley and J. R. Durrant, J. Am. Chem. Soc., 2008, 130, 3030; (b) S. Shoaee, T. M. Clarke, C. Huang, S. Barlow, S. R. Marder, M. Heeney, I. McCulloch and J. R. Durrant, J. Am. Chem. Soc., 2010, 132, 12919; (c) T. M. Clarke, A. Ballantyne, S. Shoaee, Y. W. Soon, W. Duffy, M. Heeney, I. McCulloch, J. Nelson and J. R. Durrant, Adv. Mater., 2010, 22, 5287; (d) S. Shoaee, M. P. Eng, E. Espildora, J. L. Delgado, B. Campo, N. Martin, D. Vanderzande and J. R. Durrant, Energy Environ. Sci., 2010, 3, 971.

25 A. Rao, P. C. Y. Chow, S. Gélinas, C. W. Schlenker, C.-Z. Li, H.-L. Yip, A. K.-Y. Jen, D. S. Ginger and R. H. Friend, Nature, 2013, 500, 435.

26 B. M. Savoie, A. Rao, A. A. Bakulin, S. Geélinas, B. Movaghar, R. H. Friend, T. J. Marks and M. A. Ratner, J. Am. Chem. Soc., 2014, 136, 2876. 